\title{
Sign-Consensus of Linear Multiagent Systems under a State Observer Protocol
}

\author{
Wei-Lu Diao and Cui-Qin Ma $\mathbb{D}$ \\ School of Mathematical Sciences, Qufu Normal University, Qufu 273165, Shandong, China \\ Correspondence should be addressed to Cui-Qin Ma; cuiqinma@amss.ac.cn
}

Received 26 July 2019; Accepted 18 September 2019; Published 3 November 2019

Guest Editor: Chun Wei

Copyright (C) 2019 Wei-Lu Diao and Cui-Qin Ma. This is an open access article distributed under the Creative Commons Attribution License, which permits unrestricted use, distribution, and reproduction in any medium, provided the original work is properly cited.

\begin{abstract}
The sign-consensus problem for linear time-invariant systems under signed digraph is considered. The information of the agents' states is reconstructed, and then, a state observer-type sign-consensus protocol is proposed, whose performance is analyzed using matrix analysis and ordinary differential equation theory. Sufficient conditions for ensuring sign-consensus are given. It is proven that if the adjacency matrix of the signed digraph has strong Perron-Frobenius property or is eventually positive, sign-consensus can be achieved under the proposed protocol. In particular, conventional consensus is a special case of sign-consensus under mild conditions.
\end{abstract}

\section{Introduction}

Consensus, as the key to coordination of multiagent systems (MASs), has been investigated extensively in recent years [1-9]. Most existing studies on consensus of MASs usually assume cooperative interactions among the agents, while in many cases, the agents can not only cooperate but also compete with each other, resulting in the coexistence of cooperation and competition in MASs, e.g., the two-party political system and the business alliance of competitors. To describe this scenario, the concept of bipartite consensus is proposed in [10], which means that all agents converge to the same value as required by conventional consensus but with different signs in bipartite consensus. Altafini [10] proves that, under the assumption of signed digraph being strongly connected, single-integrator MASs reach bipartite consensus if and only if the signed digraph is structurally balanced. Following this line, the strongly connected requirement on signed digraph in [10] is relaxed in [11], bipartite consensus under switched signed digraph is investigated in [12], and bipartite consensus for general linear MASs has also been studied in [13]. In [14-18], measurement noise is further considered. As pointed out in [15], the signed digraph being structurally balanced is one of the necessary and sufficient communication conditions to guarantee bipartite consensus regardless of measurement noise. This means that structural balance plays a crucial role in bipartite consensus. Structural balance is fragile and can be easily broken by changing the sign of some edge weight or by adding or deleting an edge in a signed digraph, meaning that structural unbalance can be more often seen in practice.

In fact, studies on structurally unbalanced signed digraph have already been reported in $[14,15,19-24]$, etc. In $[14,15]$, agents converge to zero in mean square under arbitrary initial conditions. In [19], the agents' states are proved to lie in between the polarized values, which are called the interval bipartite consensus. Another topic closely related to structural unbalance is the unanimity of opinion [21]; that is, all agents achieve an agreement or disagreement on a certain subject but with different extents. This concept is further extended in [23] to sign-consensus, meaning that agents reach values with the same sign but different modulus. The concept of sign-consensus is rooted in reality. For example, in a social network, people rarely have the same comment on a topic, but may have the same tendency. In the pioneering work [23], it is shown that, for the linear timeinvariant (LTI) MASs, if the graph adjacency matrix is eventually positive, sign-consensus is achieved under a state feedback sign-consensus protocol and a fully distributed sign-consensus protocol, respectively. The fixed signed 
digraph in [23] is extended in [24] to being switched over time. Sign-consensus protocols are proposed for three types of time-varying graphs, respectively. It is shown that signconsensus can be reached if the graph adjacency matrix is frequently eventually positive. We notice that both works $[23,24]$ propose state feedback-type sign-consensus protocols. However, in reality, due to constraints on measurement, it is usually hard to directly measure agents' states, but only the state estimates are available. Hence, it would be more convenient to synthesize consensus protocols based on the agents' state estimates.

With these observations, in this work, we investigate signconsensus for LTI MASs under signed digraphs. The agents' states are reconstructed, and state observer-type protocols based on them are given. By using tools from matrix analysis and ordinary differential equation theory, the closed-loop system is analyzed. It is shown that if the graph adjacency matrix has strong Perron-Frobenius property or is eventually positive, sign-consensus for LTI MASs can be achieved. Our main contributions are as follows. First, the agent dynamics are extended to be LTI system, not limited to integrators [21, 22]. Second, state observer-type consensus protocols based on state estimates between neighboring agents are proposed, which are more practical than most existing protocols based on the agents' states [23, 24].

1.1. Organization. The state observer-type consensus protocol is proposed in Section 2. The main results with respect to sign-consensus are given in Section 3. A simulation example is given to verify the proposed theoretical results in Section 4. The paper is concluded in Section 5.

1.2. Notations and Preliminaries. For a matrix or vector $A$, if all its elements are positive, $A$ is said to be positive, denoted as $A>0$. For $A \in \mathbb{R}^{n \times n}, \lambda_{i}(A)(i=1, \ldots, n)$ is the eigenvalue of $A, \operatorname{Re}\left(\lambda_{i}(A)\right)$ is the real part of $\lambda_{i}(A)$, and $\rho(A)$ is the spectral radius, which is the smallest positive real number satisfying $\rho(A) \geq\left|\lambda_{i}(A)\right|, \forall i=1, \ldots, n . \quad A \in \mathbb{R}^{n \times n}$ has the strong Perron-Frobenius property if $\rho(A)$ is a simple positive eigenvalue of $A$, and its corresponding right eigenvector $v_{r}>0$. $A \in \mathbb{R}^{n \times n}$ is eventually positive if $\exists l_{0} \in \mathbb{N}$ satisfying $A^{l}>0$, $\forall l \geq l_{0}$. For a given vector $v=\left(v_{1}, \ldots, v_{n}\right)^{T} \in \mathbb{R}^{n}, \operatorname{sign}(v)=$ $\left(\operatorname{sign}\left(v_{1}\right), \ldots, \operatorname{sign}\left(v_{n}\right)\right)^{T}$, where sign () is the sign function. $1_{n}=(1, \ldots, 1)^{T} \in \mathbb{R}^{n}, \otimes$ is the Kronecker product.

$\mathscr{G}=(\mathscr{V}, \mathcal{E}, \mathscr{F})$ is a signed digraph with $\mathscr{V}=\{1, \ldots, N\}$, $\varepsilon \subset \mathscr{V} \times \mathscr{V}$, and the adjacency matrix $\mathscr{F}=\left(f_{i j}\right) \in \mathbb{R}^{N \times N}$. $\mathcal{N}_{i}=\{(j, i) \in \mathscr{E}\}$. Generally, $\mathscr{F}=\left(f_{i j}\right) \in \mathbb{R}^{N \times N}$ is defined by $f_{i i}=0, f_{i j} \neq 0 \Longleftrightarrow(j, i) \in \varepsilon$; otherwise, $f_{i j}=0$. Denote $D_{\mathscr{F}}=\rho(\mathscr{F}) I_{N}-\mathscr{F}$ with $\rho(\mathscr{F})$ being the spectral radius of $\mathscr{F}$.

Lemma 1 (see [23]). Assume $\mathscr{G}=(\mathscr{V}, \varepsilon, \mathscr{F})$ is a signed digraph. If $\mathscr{F}$ is eventually positive, then $\mathscr{G}$ is structurally unbalanced.

Lemma 2 (see [25]). For $A \in \mathbb{R}^{n \times n}, A$ has the strong Perron-Frobenius property $\Longleftrightarrow A$ is eventually positive.

\section{Problem Formulation}

Consider an MAS with $N$ agents with each agent being described by

$$
\begin{aligned}
& \dot{x}_{i}=A x_{i}+B u_{i}, \\
& y_{i}=C x_{i}, \quad \forall i=1, \ldots, N,
\end{aligned}
$$

where $x_{i} \in \mathbb{R}^{n}, u_{i} \in \mathbb{R}^{m}$, and $y_{i} \in \mathbb{R}^{q}$ are the state, input, and output, respectively. It is assumed that $(A, B, C)$ is controllable and observable.

The communication topology among agents is represented by a signed digraph $\mathscr{G}=(\mathscr{V}, \varepsilon, \mathscr{F})$, where $\mathscr{V}=\{1, \ldots, N\}$ and $\mathscr{F}=\left(f_{i j}\right)_{N \times N}$. This paper is to synthesize a state observer-type control for each agent such that agents in (1) reach sign-consensus. Firstly, we give the definition of sign-consensus for the system in (1).

Definition 1 (see [24]). The system in (1) is said to achieve sign-consensus if there exists a consensus protocol $\left\{u_{i}, i=1, \ldots, N\right\}$ such that for any given initial states $x_{i}(0)(i=1, \ldots, N)$,

$$
\lim _{t \longrightarrow \infty}\left(\operatorname{sign}\left(x_{i}(t)\right)-\operatorname{sign}\left(x_{j}(t)\right)\right)=0, \quad i, j \in\{1, \ldots, N\} .
$$

From Definition 1, given any initial value, the states of agents in (1) converge to values with the same sign but different modulus.

Let $\bar{x}_{i}$ and $\bar{y}_{i}$ be the estimates of agent is state and output, respectively. Denote $\hat{y}_{i}=y_{i}-\bar{y}_{i}=C x_{i}-C \bar{x}_{i}$ as agent $i$ 's output estimate error. In practice, it is usually hard to measure the agent's state. Hence, it would be more convenient to design consensus protocols based on the estimates of the agents' states. The useful information for agent $i$ is synthesized by other agents' state estimates as

$$
\overline{\delta_{i}}=-\sigma_{i} \bar{x}_{i}+\sum_{j \in N_{i}} f_{i j} \bar{x}_{j}, \quad i=1, \ldots, N,
$$

where $\sigma_{i}>0$ denotes the degradation rate of the system. A state observer-type consensus protocol is given as follows:

$$
\begin{aligned}
& u_{i}=a H \overline{\delta_{i}}=a H\left(-\sigma_{i} \bar{x}_{i}+\sum_{j \in N_{i}} f_{i j} \bar{x}_{j}\right), \\
& \dot{\bar{x}}_{i}=A \bar{x}_{i}+B u_{i}-a G \hat{y}_{i}, \quad i=1, \ldots, N,
\end{aligned}
$$

where

(i) $a \in \mathbb{R} \quad$ satisfies $a \geq 1 /\left(2 \min _{i \in \Delta} \operatorname{Re}\left(\lambda_{i}\left(D_{\mathscr{F}}\right)\right)\right)$ with $\Delta=\left\{i \mid \operatorname{Re}\left(\lambda_{i}\left(D_{\mathscr{F}}\right)\right)>0, i=1, \ldots, N\right\}$;

(ii) $H$ is designed as $H=R^{-1} B^{T} P$, where $P$ is the positive definite solution of the algebraic Riccati equation

$$
A^{T} P+P A+Q-P B R^{-1} B^{T} P=0
$$

where $Q$ and $R$ are positive definite matrices;

(iii) Since $(A, C)$ is observable, $G$ can be chosen such that $A+a G C$ is Hurwitz. 
Next, we will demonstrate that the system in (1) achieves sign-consensus under the protocol in (4).

\section{Main Results}

Theorem 1. Suppose that $\mathscr{F}$ has the strong Perron-Frobenius property. Then, the system in (1) can reach sign-consensus under the protocol in (4) with $\sigma_{i}=\rho(\mathscr{F})(\forall i=1, \ldots, N)$. Moreover, the state estimate error will converge to 0 , i.e., $\lim _{t \rightarrow \infty}\left(x_{i}-\right.$ $\left.\bar{x}_{i}\right)=0$ and $\forall i=1, \ldots, N$.

Proof. Define $X=\left[x_{1}^{T}, \ldots, x_{N}^{T}\right]^{T}$ and $\bar{X}=\left[\bar{x}_{1}^{T}, \ldots, \bar{x}_{N}^{T}\right]^{T}$. Applying the protocol in (4) to the system in (1), we obtain

$$
\dot{X}=\left(I_{N} \otimes A\right) X-\left(a D_{\mathscr{F}} \otimes B H\right) \bar{X} .
$$

By direct calculation, we obtain

$$
\dot{\bar{X}}=\left(I_{N} \otimes A-a D_{\mathscr{F}} \otimes B H\right) \bar{X}-\left(a I_{N} \otimes G C\right) \hat{X},
$$

where $\hat{X}=X-\bar{X}$. Clearly,

$$
\dot{\hat{X}}=\left[I_{N} \otimes(A+a G C)\right] \widehat{X} .
$$

Since $G$ is chosen such that $A+a G C$ is Hurwitz, $I_{N} \otimes(A+a G C)$ is Hurwitz. Immediately, one has

$$
\begin{aligned}
\lim _{t \longrightarrow \infty} \widehat{X} & =0, \\
\text { i.e., } \lim _{t \rightarrow \infty}\left(x_{i}-\bar{x}_{i}\right) & =0, \quad i=1, \ldots, N .
\end{aligned}
$$

By assumption, $\mathscr{F}$ has the strong Perron-Frobenius property. This implies that $\rho(\mathscr{F})$ is a simple positive eigenvalue of $\mathscr{F}$, and its corresponding right eigenvector $v_{r}>0$. Noting that $D_{\mathscr{F}}=\rho(\mathscr{F}) I_{N}-\mathscr{F}$. Hence, $\lambda_{i}\left(D_{\mathscr{F}}\right)=\rho(\mathscr{F})-\lambda_{i}(\mathscr{F})$, $i=1, \ldots, N$. Clearly, 0 is a simple eigenvalue of $D_{\mathscr{F}}$ with corresponding right eigenvector $v_{r}$. For simplicity, we assume $\lambda_{1}\left(D_{\mathscr{F}}\right)=0$. Then, $\operatorname{Re}\left(\lambda_{i}\left(D_{\mathscr{F}}\right)\right)>0, i=2, \ldots, N$. There must exist a nonsingular matrix $\mathcal{S}=\left[v_{r} \mid S_{2}\right] \in \mathbb{R}^{N \times N}$ with $S_{2} \in \mathbb{R}^{N \times(N-1)}$ such that

$$
\mathcal{S}^{-1} D_{\mathscr{F}} \mathcal{S}=J=\left[\begin{array}{cc}
0 & 0 \\
0 & J_{N-1}
\end{array}\right],
$$

where $J_{N-1} \in \mathbb{R}^{(N-1) \times(N-1)}$ is a Jordan block with $\lambda_{i}\left(D_{\mathscr{F}}\right)(i=2, \ldots, N)$ on its diagonal. Let $W(t)=\left(\mathcal{S}^{-1} \otimes I_{n}\right)$ $X \triangleq\left[W_{1}^{T}(t), \ldots, W_{N}^{T}(t)\right]^{T}=\left[W_{1}^{T}(t) \mid \Omega^{T}(t)\right]^{T}$ and $\widehat{W}(t)=$ $\left(\mathcal{S}^{-1} \otimes I_{n}\right) \widehat{X} \triangleq\left[\widehat{W}_{1}^{T}(t)\left[\widehat{\Omega}^{T}\right](t)\right]^{T}$. Then, by (6)-(8), we have

$$
\dot{W}(t)=\left(I_{N} \otimes A-a J \otimes B H\right) W(t)+(a J \otimes B H) \widehat{W}(t),
$$

or equivalently,

$$
\begin{aligned}
\dot{W}_{1}(t) & =A W_{1}(t), \\
\dot{\Omega}(t) & =\left(I_{N-1} \otimes A-a J_{N-1} \otimes B H\right) \Omega(t)+\left(a J_{N-1} \otimes B H\right) \widehat{\Omega}(t) .
\end{aligned}
$$

Obviously, $I_{N-1} \otimes A-a J_{N-1} \otimes B H$ is an upper triangle block matrix with diagonal blocks $A-a \lambda_{i}\left(D_{\mathscr{F}}\right) B H, i=2$, $\ldots, N$. Notice that $a \geq 1 /\left(2 \min _{i \in \Delta} \operatorname{Re}\left(\lambda_{i}\left(D_{\mathscr{F}}\right)\right)\right)$ and $H=R^{-1} B^{T} P$, where $P$ is positive definite solution of (5). Then, by adopting the similar arguments as in [26], we obtain that $A-a \lambda_{i}\left(D_{\mathscr{F}}\right) B H(i=2, \ldots, N)$ is Hurwitz. By (9), we know that $\lim _{t \rightarrow \infty} \widehat{W}(t)=\lim _{t \rightarrow \infty}\left(\mathcal{S}^{-1} \otimes I_{n}\right) \hat{X}=0$, and hence, $\lim _{t \rightarrow \infty} \widehat{\Omega}(t)=0$. According to (12) and the ordinary differential equation theory, we obtain

$$
\begin{aligned}
\lim _{t \longrightarrow \infty} W_{1}(t) & =\lim _{t \longrightarrow \infty} e^{A t} W_{1}(0), \\
\lim _{t \longrightarrow \infty} \Omega(t) & =0 .
\end{aligned}
$$

$\Omega(t)$,

Since $\quad X=\left(\delta \otimes I_{n}\right) W(t)=\left(v_{r} \otimes I_{n}\right) W_{1}(t)+\left(S_{2} \otimes I_{n}\right)$

$$
\lim _{t \longrightarrow \infty} X(t)=\left(v_{r} \otimes I_{n}\right) \lim _{t \longrightarrow \infty} e^{A t} W_{1}(0),
$$

or equivalently,

$$
\lim _{t \longrightarrow \infty} x_{i}(t)=v_{r_{i}} \lim _{t \rightarrow \infty} e^{A t} W_{1}(0),
$$

where $v_{r_{i}}$ is the $i$ th component of $v_{r}$. Note that $v_{r_{i}}>0, \forall i=1, \ldots, N$. Thus, by Definition 1 , the system in (1) can achieve sign-consensus under the protocol in (4).

Remark 1. In Theorem 1, sign-consensus is achieved based on a state observer-type protocol. This is different from protocols in $[23,24]$ where state feedback protocols are proposed. Communication topology $\mathscr{G}=(\mathscr{V}, \varepsilon, \mathscr{F})$ is directed, and it is assumed that $\mathscr{F}$ has the strong PerronFrobenius property. Such an assumption together with Lemma 1 implies that $\mathscr{G}$ is structurally unbalanced.

Theorem 2. For the system in (1), suppose that $\mathscr{F}$ is eventually positive. Then, the system in (1) can achieve signconsensus under the protocol in (4) with $\sigma_{i}=\rho(\mathscr{F})$ $(\forall i=1, \ldots, N)$.

Proof. By assumption, $\mathscr{F}$ is eventually positive. This together with Lemma 2 gives that $\mathscr{F}$ has the strong Perron-Frobenius property. Applying the same procedure as in Theorem 1, one sees that the system in (1) can achieve sign-consensus under the protocol in (4).

From (14), we know that $v_{r}$ is vital to the collective behavior of MASs. In particular, if $v_{r}=1_{n}$, then conventional consensus is achieved.

Corollary 1. For the system in (1), suppose that $\mathscr{F}$ has the strong Perron-Frobenius property. Then, the system in (1) can achieve conventional consensus under the protocol in (4) with $v_{r}=1_{n}$ and $\sigma_{i}=\rho(\mathscr{F})(\forall i=1, \ldots, N)$.

Remark 2. In Corollary 1, if the requirement on $\mathscr{F}$ is changed to being eventually positive, Corollary 1 still holds.

\section{Simulation}

Example 1. Consider the system in (1) with 6 agents, where 


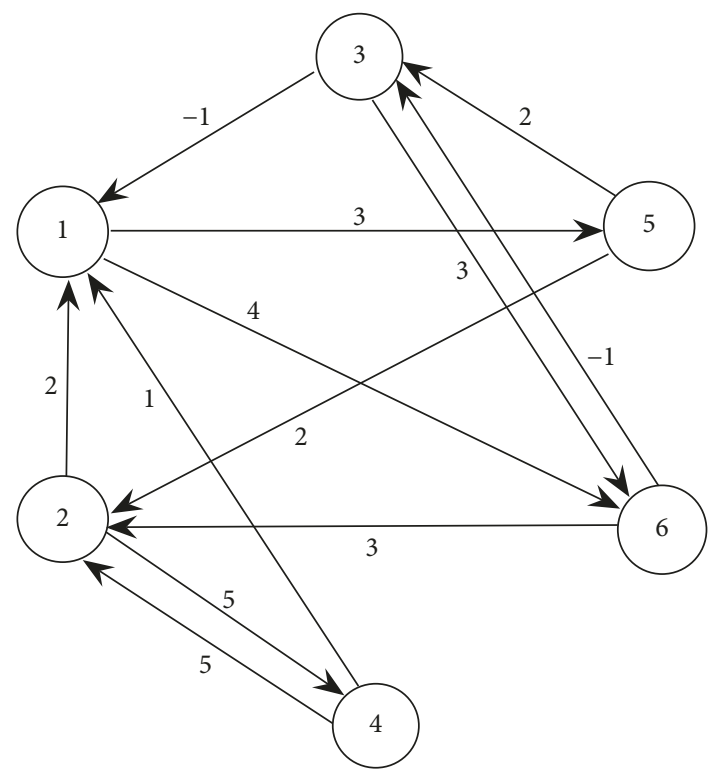

FIGURE 1: Communication topology among 6 agents.

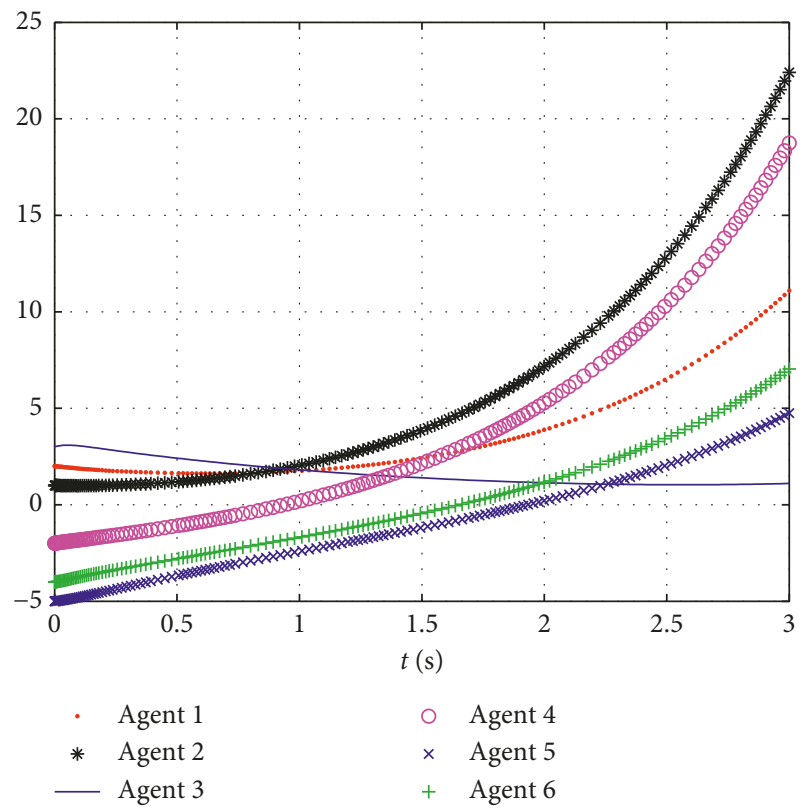

FIGURE 2: State trajectories of $x_{i 1}$ for 6 agents.

$$
\begin{aligned}
& A=\left[\begin{array}{ll}
1 & 1 \\
0 & 0
\end{array}\right], \\
& B=\left[\begin{array}{l}
0 \\
1
\end{array}\right], \\
& C=\left[\begin{array}{ll}
2 & 1 \\
0 & 1
\end{array}\right] .
\end{aligned}
$$

Obviously, $(A, B, C)$ is controllable and observable. Assume that interactions among the 6 agents are represented by $\mathscr{G}=(\mathscr{V}, \varepsilon, \mathscr{F})$, where $\mathscr{V}=\{1, \ldots, 6\}$ and $\mathscr{F}=\left(f_{i j}\right) \in \mathbb{R}^{6 \times 6}$ with $f_{12}=f_{25}=f_{35}=2, f_{13}=f_{36}=-1, f_{14}=1, f_{24}=$ $f_{42}=5, f_{26}=f_{51}=f_{63}=3$, and $f_{61}=4$. From Figure 1,

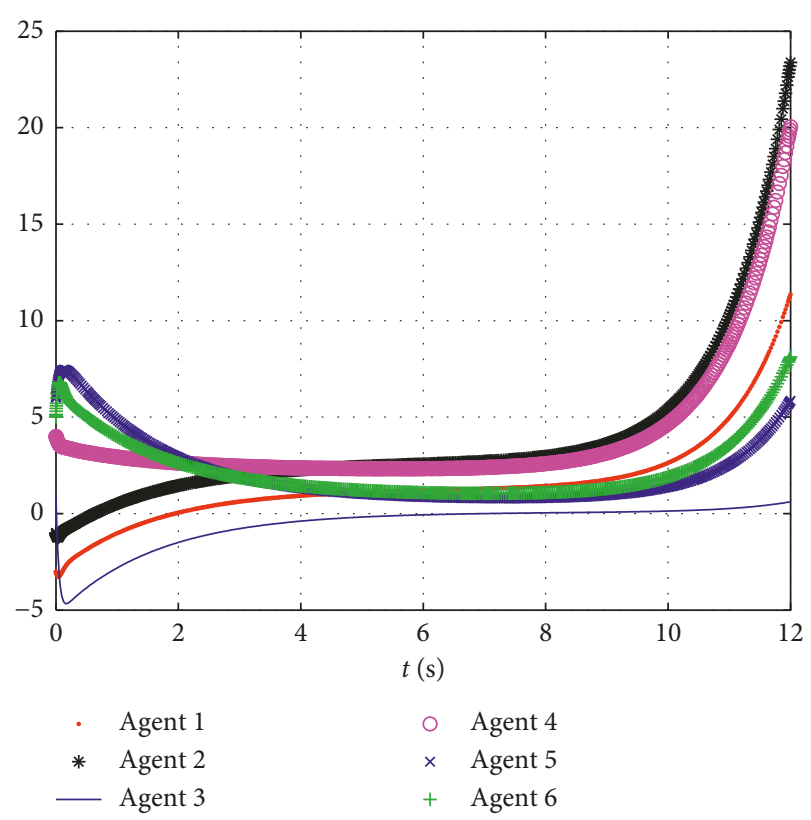

FIgURE 3: State trajectories of $x_{i 2}$ for 6 agents.

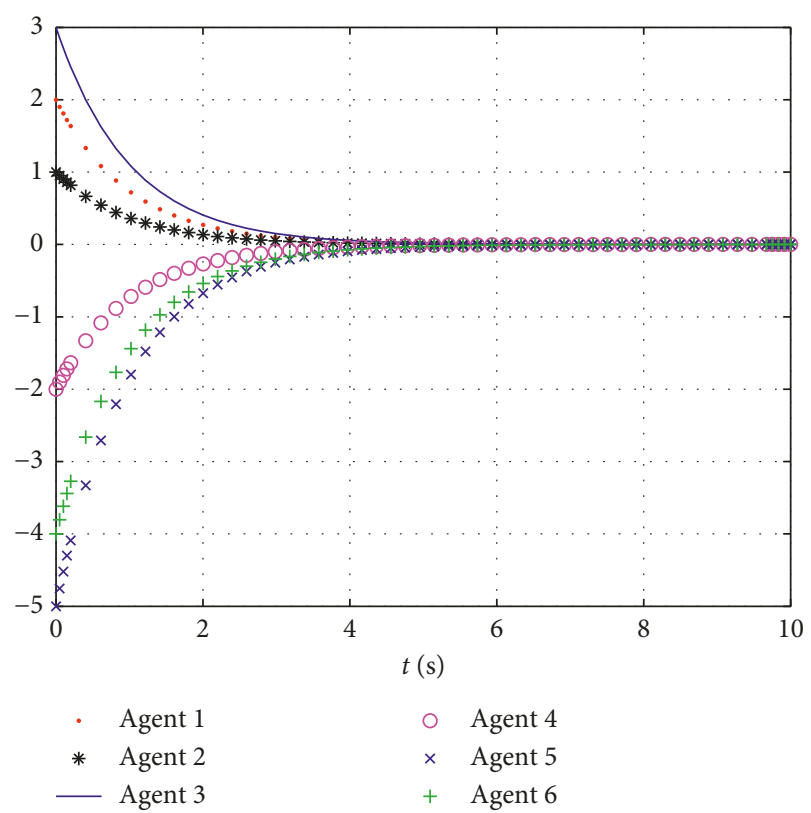

Figure 4: State estimate error trajectories of $x_{i 1}$.

$\mathscr{G}$ is structurally unbalanced. By direct calculation, one has $\mathscr{F}^{l}>0, \forall l \geq 7$. This implies that $\mathscr{F}$ is eventually positive. Therefore, by Lemma 2, one sees that $\mathscr{F}$ has strong PerronFrobenius property, and hence, communication conditions in Theorem 1 and Theorem 2 are satisfied.

Note that $\lambda_{1}\left(D_{\mathscr{F}}\right)=0, \lambda_{2}\left(D_{\mathscr{F}}\right)=10.4347, \lambda_{3}\left(D_{\mathscr{F}}\right)=$ $6.7544-1.4418 j\left(j^{2}=-1\right), \quad \lambda_{4}\left(D_{\mathscr{F}}\right)=6.7544+1.4418 j, \quad \lambda_{5}$ $\left(D_{\mathscr{F}}\right)=5.5158-2.1144 j$, and $\lambda_{6}\left(D_{\mathscr{F}}\right)=5.5158+2.1144 j$. Therefore, $\quad a \geq 1 /\left(2 \min _{2 \leq i \leq 6} \operatorname{Re}\left(\lambda_{i}\left(D_{\mathscr{F}}\right)\right)\right)=0.0906$, and hence, we assume $a=1$. Let $G=\left[\begin{array}{cc}-1 & 0 \\ 0 & -1\end{array}\right]$. By calculation, one obtains that $A+a G C$ is Hurwitz. Choose $Q=C C^{T}$ and 


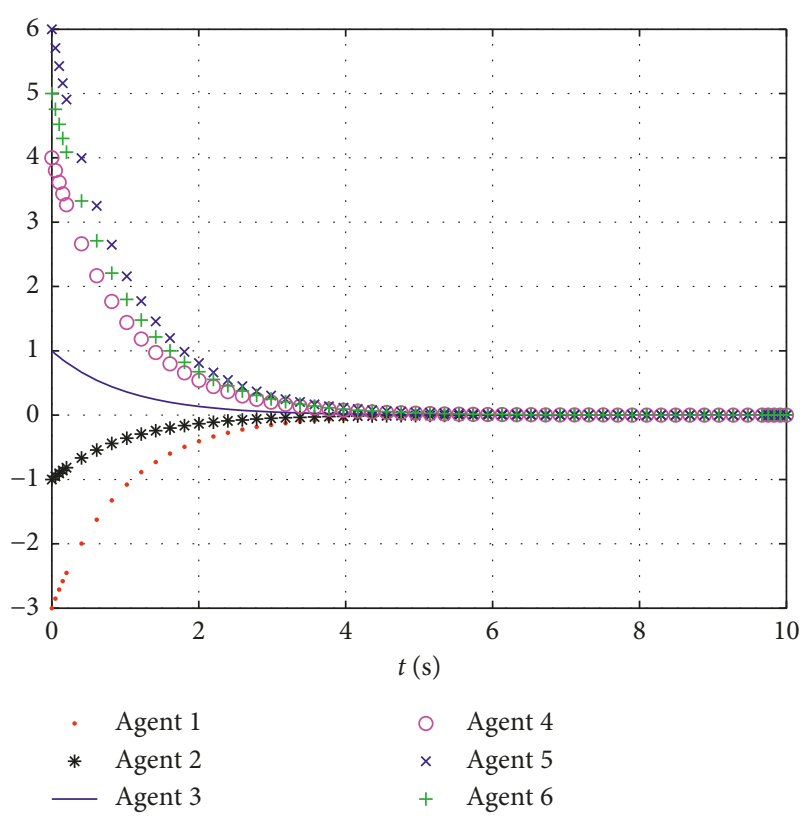

FIGURE 5: State estimate error trajectories of $x_{i 2}$.

$R=1$. Then, one can get $P=\left[\begin{array}{cc}12.3485 & 5.4495 \\ 5.4495 & 3.4495\end{array}\right]$ which is the positive definite solution of (5), and $H=R^{-1} B^{T}$ $P=[5.44953 .4495] \in \mathbb{R}^{1 \times 2}$. Applying the protocol in (4) to the system in (1), we obtain that sign-consensus is achieved. The simulation results are given in Figures 2 and 3, where $x_{i 1}$ and $x_{i 2}$ are the first and second components of agent $i$, respectively. Moreover, from Figures 4 and 5, we see that state estimate error converges to zero. Thus, the simulation example verifies the validity of Theorem 1 and Theorem 2 .

\section{Conclusions}

The sign-consensus of MASs is investigated where each agent is described by an LTI system. A state observer-type protocol is designed, which is more practical than the usual state feedback protocols. It is shown that if the adjacency matrix has strong Perron-Frobenius property or is eventually positive, then sign-consensus can be achieved based on the proposed protocols.

\section{Data Availability}

The MATLAB code used in the example can be obtained from the corresponding author.

\section{Conflicts of Interest}

The authors declare that they have no conflicts of interest.

\section{Acknowledgments}

This work was supported by the National Natural Science Foundation of China (grant no. 61973183), Natural Science Foundation of Shandong Province (grant no. ZR2019MF041), and the Youth Creative Team Sci-Tech Program of Shandong Universities (grant no. 2019KJI007).

\section{References}

[1] R. Olfati-Saber and R. M. Murray, "Consensus problems in networks of agents with switching topology and time-delays," IEEE Transactions on Automatic Control, vol. 49, no. 9, pp. 1520-1533, 2004.

[2] W. Ren and R. W. Beard, "Consensus seeking in multiagent systems under dynamically changing interaction topologies," IEEE Transactions on Automatic Control, vol. 50, no. 5, pp. 655-661, 2005.

[3] C. Q. Ma, T. Li, and J. F. Zhang, "Necessary and sufficient conditions for consensusability of linear multi-agent systems," IEEE Transactions on Automatic Control, vol. 55, no. 5, pp. 1263-1268, 2010.

[4] Z. K. Li, Z. S. Duan, G. R. Chen, and L. Huang, "Consensus of multiagent systems and synchronization of complex networks: a unified viewpoint," IEEE Transactions on Circuits and Systems, vol. 57, no. 1, pp. 213-224, 2010.

[5] C. Q. Ma, T. Li, and J. F. Zhang, "Consensus control for leader-following multi-agent systems with measurement noises," Journal of Systems Science and Complexity, vol. 23, no. 1, pp. 35-49, 2010.

[6] B. C. Wang and J. F. Zhang, "Distributed control of multiagent systems with random parameters and a major agent," Automatica, vol. 48, no. 9, pp. 2093-2106, 2012.

[7] Y. Sun, Z. Wu, and F. Meng, "Common weak linear copositive Lyapunov functions for positive switched linear systems," Complexity, vol. 2018, Article ID 1365960, 7 pages, 2018.

[8] C. Wei, M. Benosman, and T. Kim, "Online parameter identification for state of power prediction of Lithium-ion batteries in electric vehicles using extremum seeking," International Journal of Control, Automation and Systems, vol. 17, pp. 1-11, 2019.

[9] M. Xing, H. Shen, and Z. Wang, " $H_{\infty}$ synchronization of semi-markovian jump neural networks with randomly occurring time-varying delays," Complexity, vol. 2018, Article ID 8094292, 16 pages, 2018.

[10] C. Altafini, "Consensus problems on networks with antagonistic interactions," IEEE Transactions on Automatic Control, vol. 58, no. 4, pp. 935-946, 2013.

[11] J. P. Hu and W. X. Zheng, "Emergent collective behaviors on coopetition networks," Physics Letters A, vol. 378, no. 26-27, pp. 1787-1796, 2014.

[12] A. V. Proskurnikov, A. S. Matveev, and M. Cao, "Opinion dynamics in social networks with hostile camps: consensus vs. polarization," IEEE Transactions on Automatic Control, vol. 61, no. 6, pp. 1524-1536, 2016.

[13] H. Zhang and J. Chen, "Bipartite consensus of multi-agent systems over signed graphs: state feedback and output feedback control approaches," International Journal of Robust and Nonlinear Control, vol. 27, no. 1, pp. 3-14, 2017.

[14] C. Q. Ma and Z. Y. Qin, "Bipartite consensus on networks of agents with antagonistic interactions and measurement noises," IET Control Theory and Applications, vol. 10, no. 17, pp. 2306-2313, 2016.

[15] C. Q. Ma, Z. Y. Qin, and Y. B. Zhao, "Bipartite consensus of integrator multi-agent systems with measurement noise," IET Control Theory and Applications, vol. 11, no. 18, pp. 33133320, 2017.

[16] C. Q. Ma, W. W. Zhao, and Y. B. Zhao, "Bipartite linear $\chi$-consensus of double-integrator multi-agent systems with 
measurement noise," Asian Journal of Control, vol. 20, no. 1, pp. $577-584,2018$.

[17] C. Q. Ma, W. W. Zhao, and Y. B. Zhao, "Bipartite consensus of discrete-time double-integrator multi-agent systems with measurement noise," Journal of Systems Science and Complexity, vol. 31, no. 6, pp. 1525-1540, 2018.

[18] C. Q. Ma and L. H. Xie, "Necessary and sufficient conditions for leader-following bipartite consensus with measurement noise," IEEE Transactions on Systems, Man, and Cybernetics: Systems, pp. 1-6, 2018

[19] D. Meng, M. Du, and Y. Jia, "Interval bipartite consensus of networked agents associated with signed digraphs," IEEE Transactions on Automatic Control, vol. 61, no. 12, pp. 3755-3770, 2016.

[20] J. P. Hu and W. X. Zheng, "Bipartite consensus for multiagent systems on directed signed networks," in Proceedings of the 52nd IEEE Conference on Decision and Control (CDC), pp. 3451-3456, Florence, Italy, December 2013.

[21] C. Altafini and G. Lini, "Predictable dynamics of opinion forming for networks with antagonistic interactions," IEEE Transactions on Automatic Control, vol. 60, no. 2, pp. 342357, 2015.

[22] W. Xia, M. Cao, and K. H. Johansson, "Structural balance and opinion separation in trust-mistrust social networks," IEEE Transactions on Control of Network Systems, vol. 3, no. 1, pp. 46-56, 2016.

[23] Y. Jiang, H. Zhang, and J. Chen, "Sign-consensus of linear multi-agent systems over signed directed graphs," IEEE Transactions on Industrial Electronics, vol. 64, no. 6, pp. 5075-5083, 2017.

[24] Y. Jiang, H. Zhang, and J. Chen, "Sign-consensus over cooperative-antagonistic networks with switching topologies," International Journal of Robust and Nonlinear Control, vol. 28, no. 18, pp. 6146-6162, 2018.

[25] D. Noutsos, "On Perron-Frobenius property of matrices having some negative entries," Linear Algebra and Its Applications, vol. 412, no. 2-3, pp. 132-153, 2006.

[26] H. W. Zhang, F. L. Lewis, and A. Das, "Optimal design for synchronization of cooperative systems: state feedback, observer and output feedback," IEEE Transactions on Automatic Control, vol. 56, no. 8, pp. 1948-1952, 2011. 


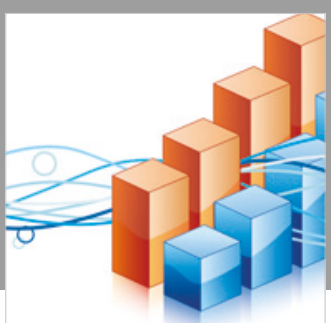

Advances in

Operations Research

\section{-n-m}
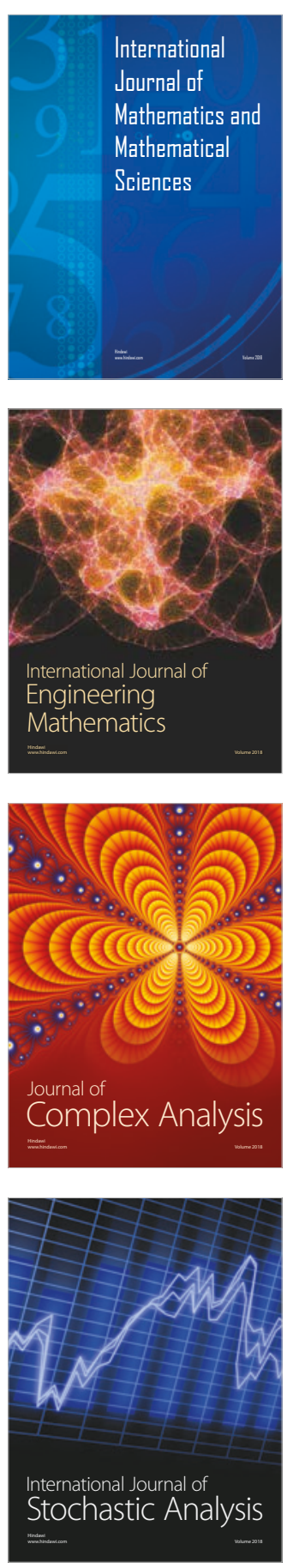
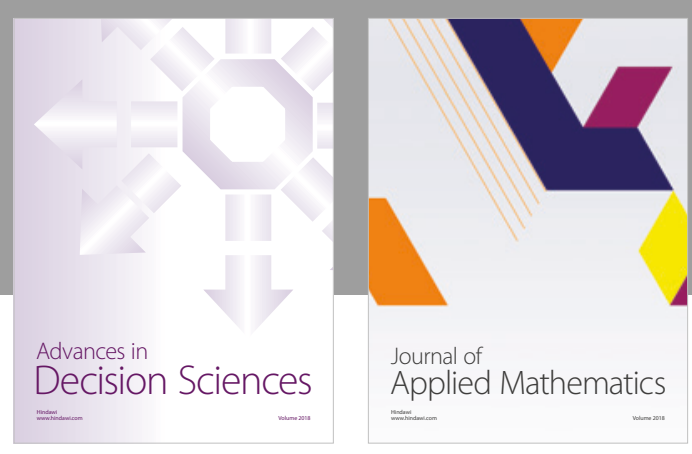

Journal of

Applied Mathematics
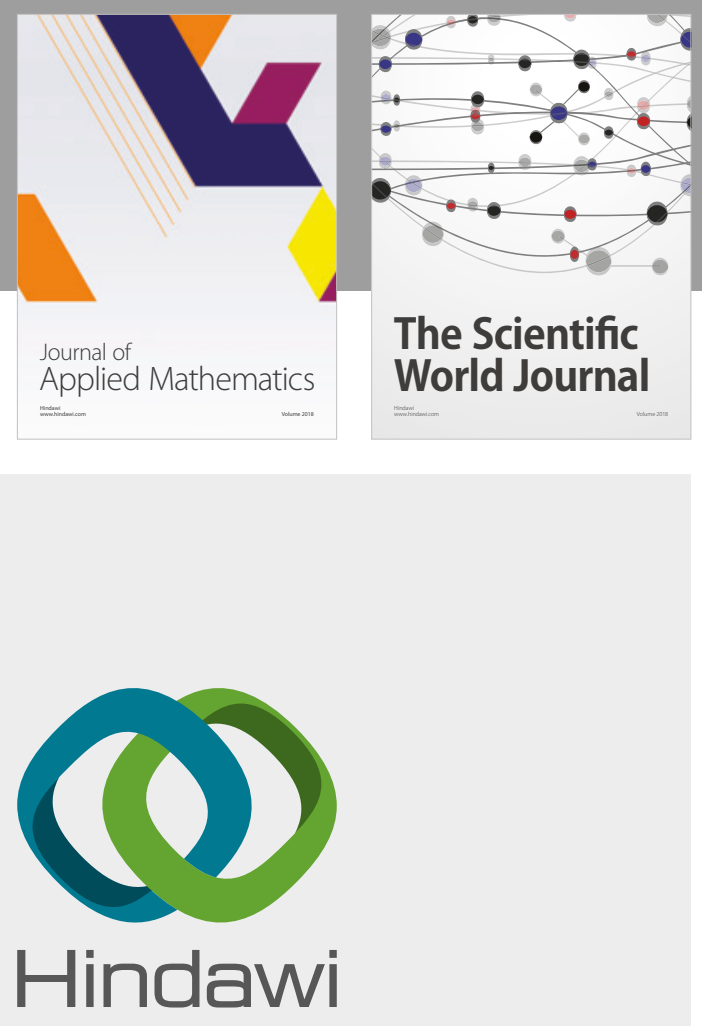

Submit your manuscripts at

www.hindawi.com

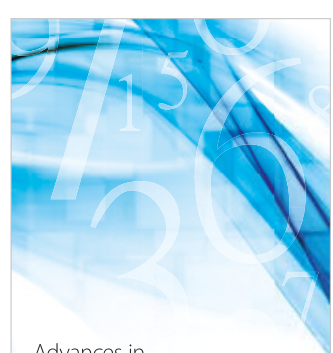

Advances in
Numerical Analysis
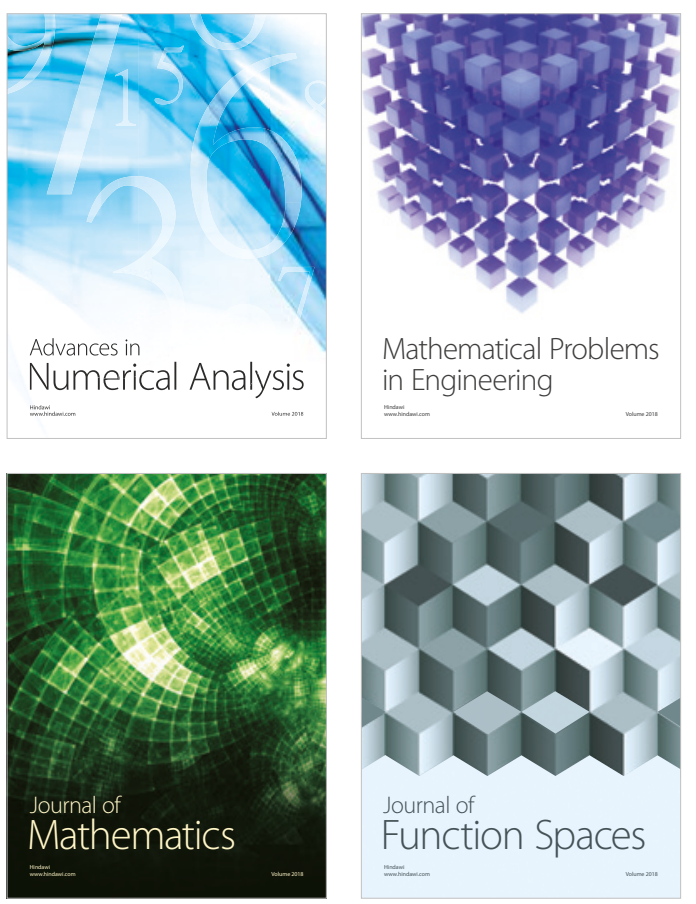

Mathematical Problems in Engineering

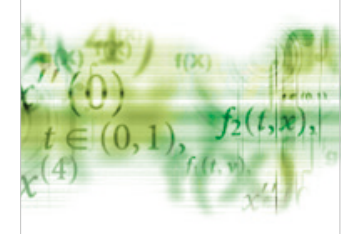

International Journal of

Differential Equations

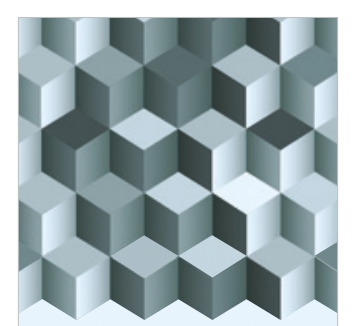

Journal of

Function Spaces
The Scientific

World Journal

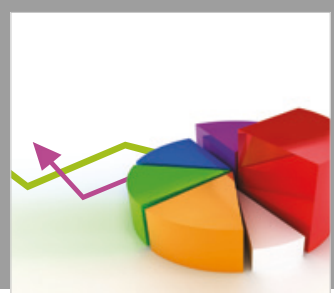

Journal of

Probability and Statistics
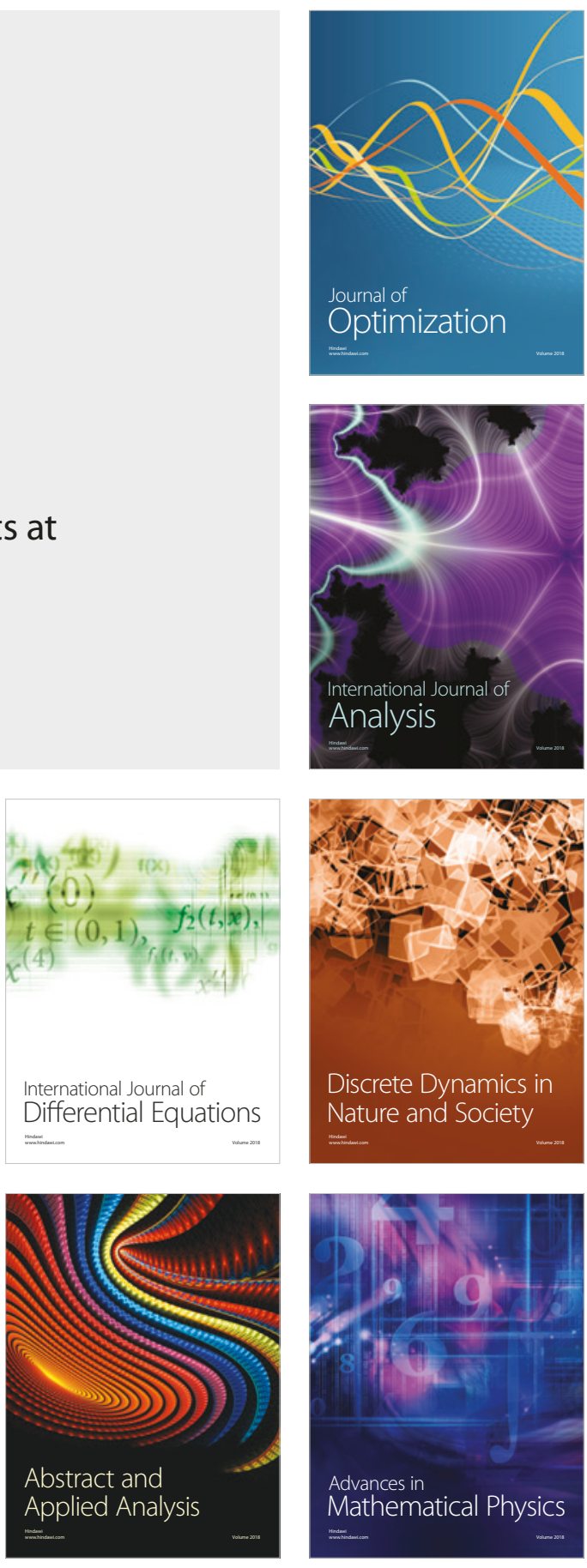